[10] B. Schweizer, Probabilistio metric spaces - the first 25 years, The New York Statistician 19 (1967), pp. 3-6.

[11] - Multiplications on the space of probability distribution functions, Aequationes Math. 12 (1975), pp. 156-183.

[12] B. Schweizer and A. Sklar, Operations on distribution functions not derivable from operations on random variables, Studia Math. 52 (1974), pp. 43-52.

[13] A. N. Serstne $\mathrm{Y}$, The notion of a random normed space, Dokl. Acad. Nauk SSSR 149 (1963), pp. 280-283 [translated in Soviet Math. Dold. 4 (1963), pp. 388-391].

[14] D. A. Sibley, A metric for wealc convergenee of distribution functions, Rocky Mountain J. Math. 1 (1971), pp. 427-430.

[15] R. A. Wijeman, Oonvergence of sequences of convex sets, cones and functions II, Trans. Amer. Math. Soc. 123 (1966), pp. 32-45.

[16] S. Willard, General topology, Addison-Wesley, Reading, Mass. 1970.

[17] R. Moynihan, Infinite $\tau_{T}$ products of distribution funotions, J. Austral. Math. Soc. (Series A) 26 (1978), pp. 227-240.

\section{ANALYSIS DEPARTMANT}

Bedford, Massachusetts \\ STUDIA MATHEMATICA, T. LXIX. (1980)}

Weighted inequalities for vector-valued maximal functions and singular integrals

by

KENNETH F. ANDERSEN (Edmonton, Alta) and RUSSEL T. JOHN (Holmdel, N. J.)

Abstract. Weighted weak and strong type norm inequalities are derived for a vector-valued analogue of the Hardy-Littlewood maximal function operator and these in turn are used to obtain weighted inequalities for the classical Marcinkiewicz integral and a wide class of singular integral operators defined on $\boldsymbol{R}^{n}$.

\$1. Introduction. The Hardy-Littlewood maximal function $f^{*}(x)$ is defined for locally integrable functions $f$ on $\boldsymbol{R}^{n}$ by

$$
f^{*}(x)=\sup \frac{1}{|Q|} \int_{Q}|f(y)| d y \quad\left(x \in \boldsymbol{R}^{n}\right),
$$

the supremum being taken over all cubes $Q$ of Lebesgue measure $|Q|$, centered at $x$ with sides parallel to the co-ordinate axis. The operator $M: f \rightarrow f^{*}$ and its variants have been widely studied, in particular, the well-known inequalities

$$
\begin{gathered}
\int_{\boldsymbol{R}^{n}}\left|f^{*}(x)\right|^{p} d x \leqslant O_{p} \int_{\boldsymbol{R}^{n}}|f(x)|^{p} d x \quad(1<p<\infty), \\
\left|\left\{x \in \boldsymbol{R}^{n}: f^{*}(x)>\alpha\right\}\right| \leqslant C \alpha^{-1} \int_{\boldsymbol{R}^{n}}|f(x)| d x \quad(\forall a>0), \\
\text { ess } \sup _{x \in \boldsymbol{R}^{n}} f^{*}(x) \leqslant \operatorname{ess} \sup _{x \in \boldsymbol{R}^{n}}|f(x)|
\end{gathered}
$$

have been generalized and extended in various directions.

Let $\omega(x)$ be non-negative, locally integrable on $\boldsymbol{R}^{n}$ and for measurable $E \subset \boldsymbol{R}^{n}$ put $\omega(E)=\int \omega(x) d x$. We say that $\omega \in A_{p},(1 \leqslant p<\infty)$ if there is a constant $K$ such that

$$
\left(\frac{1}{|Q|} \int_{Q} \omega(x) d x\right)\left(\frac{1}{|Q|} \int_{Q} \omega(x)^{-1 /(p-1)} d x\right)^{p-1} \leqslant K
$$


for all cubes $Q \subset \boldsymbol{R}^{n}$. In (1.4), products of the form $0 \cdot \infty$ are taken to be zero, and for $p=1$ the second factor on the left is understood to be ess sup $\omega(x)^{-1}$. In [13] B. Muckenhoupt proved that $\omega \in A_{p}$ is both necess$x \in Q$ ary and sufficient in order that the following weighted analogues of (1.1) and (1.2) should hold:

$$
\int_{\mathbf{R}^{n}}\left|f^{*}(x)\right|^{p} \omega(x) d x \leqslant C_{p} \int_{\boldsymbol{n}^{n}}|f(x)|^{p} \omega(x) d x \quad(1<p<\infty),
$$

$$
\omega\left(\left\{x \in \boldsymbol{R}^{n}: f^{*}(x)>\alpha\right\}\right) \leqslant 0_{p} a^{-p} \int_{\boldsymbol{R}^{n}}|f(x)|^{p} \omega(x) d x \quad(1 \leqslant p<\infty) .
$$

These results were used later by R. Eunt, B. Muckenhoupt and R. Wheeden [9] and by R. Coifman and $O$. Fefferman [5] to obtain weighted norm inequalities for Hilbert transforms in $\boldsymbol{R}^{1}$ and singular integrals in $\boldsymbol{R}^{n}$, respectively.

In a different direction C. Fefferman and E. Stein [7] obtained vector-valued analogues of (1.1)-(1.3) and applied these to obtain certain estimates for the Marcinkiewicz integral. If $f=\left\{f_{k}\right\}_{1}^{\infty}$, is a sequence of locally integrable functions on $\boldsymbol{R}^{n}, f^{*}=\left\{f_{k}^{*}\right\}_{1}^{\infty}$, and $|f(x)|_{r}=\left(\sum_{k=1}^{\infty}\left|f_{k}(x)\right|^{r}\right)^{1 / r}$, $1<r<\infty$, their main result may be stated as follows:

$$
\begin{gathered}
\int_{\boldsymbol{R}^{n}}\left|f^{*}(x)\right|_{r}^{p} d x \leqslant C_{r, p} \int_{\boldsymbol{n}^{n}}|f(x)|_{r}^{p} d x \quad(1<p<\infty), \\
\left|\left\{x \in \boldsymbol{R}^{n}:\left|f^{*}(x)\right|_{r}>\alpha\right\}\right| \leqslant C_{r, p} \alpha^{-p} \int_{\boldsymbol{R}^{n}}|f(x)|_{r}^{p} d x \quad(1 \leqslant p<\infty) .
\end{gathered}
$$

(1.9) If $|E|<\infty$ and $|f(x)|_{r}$ is bounded and supported on $E$, then $\left|f^{*}(x)\right|_{r}^{r} \in \exp L(E)$.

The purpose of this paper is to obtain the weighted analogues of (1.7)-(1.9), the corresponding weighted estimates for the Marcinkiewicz integral and weighted norm inequalities for a wide class of vector-valued singular integral operators. Section 3 is devoted to the statement and proof of results for the vector-valued maximal function operator; tho application to the Marcinkiewicz integral is given in Section 4 while Section 5 contains the results for vector-valued singular integrals. Section 2 contains the statement of two interpolation lemmas as well as several facts about the $A_{p}$ condition which are required in the sequel.

As usual $C_{p}, \ldots$ will denote an absolute constant, not necessarily the same at each occurrence, depending only on $n, \omega$ and the parameters indicated by subscripts.

\$ 2. Preliminaries. In addition to the $A_{p}$ condition already defined for $1 \leqslant p<\infty$ we shall require also the $A_{\infty}$ condition, namely $\omega \in A_{\infty}$ if there are positive constants $K, \delta$ such that

$$
\frac{\omega(E)}{\omega(Q)} \leqslant K\left(\frac{|E|}{|Q|}\right)^{\delta}
$$

for every cube $Q$ and measurable $E \subset Q$.

The following properties, used frequently in the sequel, are stated here for easy reference. For proofs, see [13], [5], [9] and [14].

$$
\begin{aligned}
& \omega(x) \in A_{p} \Leftrightarrow \omega(x)^{1-p^{\prime}} \in A_{p^{\prime}}, \quad 1<p<\infty, 1 / p+1 / p^{\prime}=1, \\
& \omega(x) \in A_{p} \Rightarrow \omega(x) \in A_{q} \forall q>p, \\
& \omega(x) \in A_{p},(p>1) \Rightarrow \omega(x) \in A_{q} \text { for some } q<p, \\
& \omega(x) \in A_{\infty} \Leftrightarrow \omega(x) \in A_{p} \text { for some } p \geqslant 1,
\end{aligned}
$$

$$
\begin{aligned}
\omega(x) \in A_{p} \Rightarrow & \omega(x)>0 \text { a.e. and } \omega(x)^{q} \text { is locally integrable for } \\
& \text { some } q>1, \\
\omega(x) \in A_{p} \Rightarrow & \omega(\bar{Q}) \leqslant O \omega(Q) \text { for all cubes } Q \text { where } \bar{Q} \text { is the cube }
\end{aligned}
$$
concentric with $Q$ and diameter $(\bar{Q})=2$ diameter $(Q)$.

The following interpolation results will be required. The first is essentially the Marcinkiewicz interpolation Theorem, see [1]. The second is a vector-valued analogue of the Riesz convexity Theorem; for linear operators it is proved in [2] while the extension to the sublinear case may be patterned along the line of proof given in [4] for the scalar-valued case.

Let $S$ denote the linear space of sequences $f=\left\{f_{k}\right\}$ of the form: $f_{k}(\boldsymbol{x})$ is a simple function on $\boldsymbol{R}^{n}$ and $f_{k}(x) \equiv \mathbf{0}$ for all sufficiently large $k$. $S$ is dense in $L_{\omega}^{p}\left(l^{r}\right), 1 \leqslant p, r<\infty$, see [2].

LeimMa 2.1. Let $\omega(x) \geqslant 0$ be locally integrable on $\boldsymbol{R}^{n}, 1<r<\infty$, $1 \leqslant p_{i} \leqslant q_{i}<\infty$ and suppose $T$ is a sublinear operator defined on $\mathbf{S}$ satisfying

$$
\omega\left(\left\{x \in \boldsymbol{R}^{n}:|T f(x)|_{r}>\alpha\right\}\right) \leqslant M_{i}^{q_{i}} \alpha^{-q_{i}}\left(\int_{\boldsymbol{R}^{n}}|f(x)|_{r}^{p_{i}} \omega(x) d x\right)^{q_{i} \mid p_{i}}
$$

for $i=0,1$ and $f \in S$. Then $T$ extends uniquely to a sublinear operator on $L_{\omega}^{p}\left(l^{r}\right)$ and there is a constant $M_{\theta}$ such that

$$
\left(\int_{\boldsymbol{R}^{n}}|T f(x)|_{r}^{q} \omega(x) d x\right)^{1 / q} \leqslant M_{\theta}\left(\int_{\boldsymbol{R}^{n}}|f(x)|_{r}^{p} \omega(x) d x\right)^{1 / \rho}
$$

where

$$
(1 / p, 1 / q)=(1-\theta)\left(1 / p_{0}, 1 / q_{0}\right)+\theta\left(1 / p_{1}, 1 / q_{1}\right), \quad 0<\theta<1 .
$$

LEMMA 2.2. Let $\omega(x) \geqslant 0$ be locally integrable on $\boldsymbol{R}^{n}, 1<r_{i}, s_{i}<\infty$, $1 \leqslant p_{i}, q_{i}<\infty$ and suppose $T$ is a sublinear operator defined on $S$ satisfying

$$
\left(\int_{\boldsymbol{R}^{n}}|T f(x)|_{s_{i}}^{q_{i}} \omega(x) d x\right)^{1 / q_{i}} \leqslant M_{i}\left(\int_{\boldsymbol{R}^{n}}|f(x)|_{r_{i}}^{p_{i}} \omega(x) d x\right)^{1 / p_{i}}
$$


for $i=0,1$ and $f \in S$. Then $T$ extends uniquely to a sublinear operator on $L_{\omega}^{p}\left(l^{r}\right)$ such that

$$
\left(\left.\int_{\boldsymbol{R}^{n}}|T f(x)|_{s}^{q} \omega(x) d x\right|^{1 / q} \leqslant M_{0}^{1-\theta} M_{1}^{\theta}\left(\int_{\boldsymbol{R}^{n}}|f(x)|_{r}^{p} \omega(x) d x\right)^{1 / p}\right.
$$

where

$$
\begin{aligned}
(1 / p, 1 / q, 1 / s, 1 / r)=(1-\theta) & \left(1 / p_{0}, 1 / q_{0}, 1 / s_{0}, 1 / r_{0}\right)+ \\
& +\theta\left(1 / p_{1}, 1 / q_{1}, 1 / s_{1}, 1 / r_{1}\right), \quad 0 \leqslant \theta \leqslant 1 .
\end{aligned}
$$

\$ 3. Maximal function inequalities. In this section we prove tho following theorem.

Temorem 3.1. Let $1<r<\infty$ and suppose $E \subset \boldsymbol{R}^{n}$ with $|E|<\infty$.

(a) If $1 \leqslant p<\infty$, there is a constant $C_{r, p}$ such that

$$
\omega\left(\left\{x \in \boldsymbol{R}^{n}:\left|f^{*}(x)\right|_{r}>\alpha\right\}\right) \leqslant O_{\alpha, p} \alpha^{-p} \int_{\boldsymbol{R}^{n}}|f(x)|_{r}^{p} \omega(x) d x
$$

if and only if $\omega \in A_{p}$.

(b) If $1<p<\infty$, there is a constant $O_{r, p}$ such that

$$
\int_{\boldsymbol{R}^{n}}\left|f^{*}(x)\right|_{r}^{p} \omega(x) d x \leqslant C_{r, p} \int_{\boldsymbol{R}^{n}}|f(x)|_{r}^{p} \omega(x) d x
$$

if and only if $\omega \in A_{p}$.

(c) If $\omega(x)^{\alpha}$ is integrable on $\mathbb{D}$ for some $q>1$, in particular if $\omega \in A_{\infty}$ and $B$ is a oube, then $\left|f^{*}(x)\right|_{r}^{r} \in \exp L_{\omega}(E)$ whenever $|f(x)|_{r}$ is bounded and supported on $E$.

H. Heinig [8] has recently obtained (a) in the case $p=1$ and has also given some results for $p$ in the range $0<p<1$. The weights considered there satisfy the condition $\omega^{*}(x) \leqslant K \omega(x)$ a.e. which is readily seen to be equivalent to the $A_{1}$ condition.

For the proof of Theorem 3.1 we require the following duality result of C. Fefferman and E. Stein [7], Lemma 1.

LEMCMA 1. Let $f, p$ be non-negative real-valued functions on $\boldsymbol{R}^{n}$ and suppose $r>1$. There exists a constant $C_{r}$, independent of $f, \varphi$ such that

$$
\int_{\boldsymbol{R}^{n}}\left|f^{*}(x)\right|^{r} \varphi(x) d x \leqslant O_{r} \int_{\boldsymbol{R}^{n}}|f(x)|^{r} \varphi^{*}(x) d x
$$

Proof of Theorem 3.1. We shall prove (a) and (b) first, then (c).

Concerning the necessity of $\omega \in A_{p}$, there is nothing to show since $\omega \in A_{x}$ is already necessary in the scalar-valued case, $f=\left\{f_{k}\right\}, f_{k}(x)=0$, $k=2,3, \ldots$ according to Muckenhoupt's results (1.5) and (1.6).

The sufficiency of $\omega \in A_{p}$ for (a) and (b) will be achieved as follows. We first obtain (3.2) for $p=r$ as an easy consequence of (1.5); then (3.1) is derived for $r>p$ and (3.2) is then obtained for $r>p$ by an appeal to Lemma 2.1. A duality argument then yields (3.2) for $r<p$ and $r$ suf- ficiently small; finally (3.2) follows for all $r<p$ by an application of Lemma 2.2. Since (3.2) always implies (3.1), the proof of (a) and (b) will be complete.

Observe first that (3.2) for the case $r=p$ is an easy consequence of (1.5) since

$$
\begin{aligned}
\int_{\boldsymbol{R}^{n}}\left|f^{*}(x)\right|_{r}^{r} \omega(x) d x & =\sum \int_{\boldsymbol{R}^{n}}\left|f_{k}^{*}(x)\right|^{r} \omega(x) d x \leqslant C_{r} \sum \int_{\boldsymbol{R}^{n}}\left|f_{k}(x)\right|^{r} \omega(x) d x \\
& =C_{r} \int_{\boldsymbol{R}^{n}}|f(x)|_{r}^{r} \omega(x) d x .
\end{aligned}
$$

Now suppose $r>p, \omega \in A_{p}$ and $\alpha>0$. As usual, we can assume withou loss of generality that $f \in S$, for then the general case follows by a standard limiting argument. The Calderón-Zygmund decomposition [15], pp. 17-18, yields a sequence of non-overlapping cubes $\left\{Q_{j}\right\}$ such that

$$
\begin{gathered}
|f(x)|_{r} \leqslant \alpha, \quad x \notin \Omega=\bigcup_{1}^{\infty} Q_{j}, \\
\alpha<\frac{1}{\left|Q_{j}\right|} \int_{Q_{j}}|f(x)|_{r} d x \leqslant 2^{n} \alpha, \quad j=1,2, \ldots
\end{gathered}
$$

Let $f=f^{\prime}+f^{\prime \prime}$ where $f^{\prime}=\left\{f_{k}^{\prime}\right\}, f_{k}^{\prime}(x)=f_{k}(x) \chi_{\boldsymbol{R}^{n_{-}}}(x)$. Minkowski's inequality shows that

$$
\left|f^{*}(x)\right|_{r} \leqslant\left|f^{\prime *}(x)\right|_{r}+\left|f^{\prime \prime *}(x)\right|_{r}
$$

so that (3.1) will follow if we show that

$$
\omega\left(\left\{x \in \boldsymbol{R}^{n}:\left|f^{\prime *}(x)\right|_{r}>\alpha\right\}\right) \leqslant C_{r, p} a^{-p} \int_{\boldsymbol{R}^{n}}|f(x)|_{r}^{p} \omega(x) d x
$$

and

$$
\text { (3.8) } \quad \omega\left(\left\{x \in \boldsymbol{R}^{n}:\left|f^{\prime \prime *}(x)\right|_{r}>\alpha\right\}\right) \leqslant \sigma_{r, p} \alpha^{-p} \int_{\mathbf{R}^{n}}|f(x)|_{r}^{p} \omega(x) d x .
$$

Since $\omega \in A_{r}$ by (2.2), (3.4) holds with $f^{\prime}$ in place of $f$, and hence the Chebysher inequality yields

$$
\omega\left(\left\{x \in \boldsymbol{R}^{n}:\left|f^{\prime *}(x)\right|_{r}>\alpha\right\}\right) \leqslant C_{r} a^{-r} \int_{\boldsymbol{R}^{n}}\left|f^{\prime}(x)\right|_{r}^{r} \omega(x) d x
$$

and since, by (3.5), $\left|f^{\prime}(x)\right|_{r}^{r} \leqslant \alpha^{r-p}\left|f^{\prime}(x)\right|_{r}^{p}$, we obtain (3.7). To prove (3.8), define $\bar{f}=\left\{\bar{f}_{k}\right\}$ by

$$
\bar{f}_{k}(x)= \begin{cases}\frac{1}{\left|Q_{j}\right|} \int_{Q_{j}}\left|f_{k}(y)\right| d y, & x \in Q_{j}, j=1,2, \ldots, \\ 0 & \text { otherwise }\end{cases}
$$

and let $\bar{Q}_{j}$ denote the cube with the same center as $Q_{j}$ but with diameter $\left(\bar{Q}_{j}\right)=(2 n)$ diameter $\left(\bar{Q}_{j}\right)$. It is shown in [7] that $f_{k}^{\prime \prime *}(x) \leqslant C \bar{f}_{k}^{*}(x)$ for 
$x \notin \bar{\Omega}=\bigcup_{1}^{\infty} \bar{Q}_{j}$. Thus (3.8) will follow if we show

$$
\omega(\bar{\Omega}) \leqslant C \alpha^{-p} \int_{\boldsymbol{R}^{n}}|f(x)|_{r}^{p} \omega(x) d x
$$

and

$$
\omega\left(\left\{x \in \boldsymbol{R}^{n}:\left|\bar{f}^{*}(x)\right|_{r}>a\right\}\right) \leqslant O_{r} a^{-p} \int_{\boldsymbol{n}^{n}}|f(x)|_{r}^{p} \omega(x) d x .
$$

If $p>1$, Eölder's inequality applied to (3.6) shows

$$
\begin{aligned}
\omega\left(Q_{j}\right) & =\int_{Q_{j}} \omega(x) d x \leqslant \alpha^{-p} \frac{1}{\left|Q_{j}\right|^{p}}\left(\int_{Q_{j}}|f(x)|_{r} d x\right)^{p} \int_{Q_{j}} \omega(x) d x \\
& \leqslant \alpha^{-p}\left(\int_{Q_{j}}|f(x)|_{r}^{p} \omega(x) d x\right)\left(\frac{1}{\left|Q_{j}\right|} \int_{Q_{j}} \omega(x)^{-1 /(p-1)} d x\right)^{p-1}\left(\frac{1}{\left|Q_{j}\right|} \int_{Q_{j}} \omega(x) d x\right)
\end{aligned}
$$

and since $\omega \in A_{p}$ we obtain

$$
\omega\left(Q_{j}\right) \leqslant C \alpha^{-p} \int_{Q_{j}}|f(x)|_{r}^{p} \omega(x) d x .
$$

A similar argument shows that (3.11) holds also if $p=1$. Using (2.4) we see that $\omega\left(\bar{Q}_{j}\right) \leqslant C \omega\left(Q_{j}\right)$ and hence (3.9) follows from (3.11) upon summing over $j$. Now Minkowski's inequality shows, by virtue of (3.6), that $|\bar{f}(x)|_{r} \leqslant 2^{n} a$, and since $|\bar{f}(x)|_{r}$ is supported in $\Omega$ we obtain in a manner similar to the proof of (3.7)

$$
\omega\left(\left\{x \in \boldsymbol{R}:\left|\bar{f}^{*}(x)\right|_{r}>\alpha\right\}\right) \leqslant O_{r} \alpha^{-r} \int_{\boldsymbol{R}^{n}}|\bar{f}(x)|_{r}^{r} \omega(x) d x \leqslant O_{r} \int_{\Omega} \omega(x) d x
$$

which together with (3.11) yields (3.10) as required. This completes the proof of (3.1) in the case $r \geqslant p$. If $r>p>1,(2.2)$ shows that for $\omega \in A_{p}$, (3.1) holds with $p$ replaced by $p_{1}$ and $p_{2}$ where $p_{1}<p<p_{2}<r$, hence Lemma 2.1 yields (3.2) for $r>p>1$.

Suppose now that $p>r$ and $\omega \in A_{p}$. By (2.2) there is an $r_{0}, 1<r_{0}<p$ such that $\omega \in A_{q}, q \geqslant p / r_{0}$. In particular, (2.1) and (2.3) yield $\omega(x)>0$ a.e. and $\omega(x)^{1-q^{\prime}} \in A_{q^{\prime}}$ so that by (1.5), if $\varphi \geqslant 0$ belongs to the unit ball of $L_{\omega}^{\alpha^{\prime}}\left(\boldsymbol{R}^{n}\right)$, then

$$
\int_{\boldsymbol{R}^{n}}\left|(\varphi \omega)^{*}(x)\right|^{\alpha^{\prime}} \omega(x)^{1-\alpha^{\prime}} d x \leqslant O_{\alpha} \int_{\boldsymbol{R}^{n}}|\varphi(x)|^{q^{\prime}} \omega(x) d x=C_{\alpha},
$$

and hence Lemma 3.1 and Hölder's inequality shows

(3.12) $\int_{\mathbf{R}^{n}}\left|f^{*}(x)\right|_{r}^{r} \varphi(x) \omega(x) d x \leqslant C_{r} \int_{\mathbf{R}^{n}}|f(x)|_{r}^{r}\left[(\varphi \omega)^{*}(x) / \omega(x)\right] \omega(x) d x$

$$
\leqslant C_{r, q}\left(\int_{\boldsymbol{R}^{n}}|f(x)|_{r}^{r q} \omega(x) d x\right)^{1 / q}
$$

Taking the supremum in (3.12) over such $\varphi$ then yields (3.2) for $1<r \leqslant r_{0}$ upon taking $q=p / r$, and this together with the case $p=r$ proved in (3.4) yields (3.2) for $r_{0}<r<p$ by an application of Lemma 2.2. The proof of (b) and with it also (a), is complete.

Finally we prove (c). Observe first that the hypothesis on $\omega$ and Hölder's inequality shows

$$
\int_{E} e^{\delta|\psi(x)|} \omega(x) d x \leqslant\left(\int_{E} e^{\delta q^{\prime}|\varphi(x)|} d x\right)^{1 / q^{\prime}}\left(\int_{E} \omega(x)^{q} d x\right)^{1 / q}
$$

so that $\|\psi\|_{\exp L_{\omega}} \leqslant O\|\psi\|_{\exp L}$. Hence

$$
\begin{aligned}
\|\varphi \omega\|_{L \ln L} & =\sup \left\{\int_{E}|\varphi(x) \psi(x)| \omega(x) d x:\|\psi\|_{\exp L} \leqslant 1\right\} \\
& \leqslant\|\varphi\|_{L_{\omega} \ln L} \sup \left\{\|\psi\|_{\exp L_{\omega}}:\|\psi\|_{\exp L} \leqslant 1\right\} \leqslant C\|\varphi\|_{L_{\omega} \ln L} .
\end{aligned}
$$

Now if $\varphi(x) \geqslant 0,|f(x)|_{r}$ are supported on $E$ and $|f(x)|_{r}$ is bounded, Lemma 3.1 yields

$$
\begin{aligned}
\int_{E}\left|f^{*}(x)\right|_{r}^{r} \varphi(x) \omega(x) d x & \leqslant C_{r} \int_{E}|f(x)|_{r}^{r}(\varphi \omega)^{*}(x) d x \\
& \leqslant O_{r}\left(\underset{x \in E}{\operatorname{esssup}}|f(x)|_{r}^{r}\right) \int_{E}(\varphi \omega)^{*}(x) d x,
\end{aligned}
$$

and by a well-known result ([15], [17]) this last integral is bounded by $O\|\varphi \omega\|_{L \ln L}$, and hence from (3.13)

$$
\int_{E}\left|f^{*}(x)\right|_{r}^{r} \varphi(x) \omega(x) d x \leqslant C_{r}\left(\operatorname{ess} \sup |f(x)|_{r}^{r}\right)\|\varphi\|_{L_{\omega} \ln L} .
$$

Taking the supremum in (3.14) over $\varphi$ in the unit ball of $L_{\omega} \ln L$ we obtain

$$
\left\|\left.f^{*}(x)\right|_{r} ^{r}\right\|_{\exp L_{\omega}} \leqslant C_{r}\left(\operatorname{ess} \sup |f(x)|_{r}^{r}\right)
$$

which gives (c). The Theorem is proved.

\$4. Application to Marcinkiewicz integrals. Following C. Fefferman and E. Stein [7] we can apply Theorem 3.1 to obtain new results for the Marcinkiewicz integral $H_{\lambda}^{\prime}$ corresponding to a disjoint collection of cubes $\left\{Q_{j}\right\}$. If $d_{j}$ is the diameter of $Q_{j}$ and $y_{j}$ its center, then $H_{\lambda}^{\prime}$ is equivalent to $\mathbb{S}_{\lambda}$ :

$$
S_{\lambda}(x)=\sum_{j=1}^{\infty} \frac{d_{j}^{n+\lambda}}{\left|x-y_{j}\right|^{n+\lambda}+d_{j}^{n+\lambda}}
$$

see [16], $\S \S 4,5$. On the otherhand, if $\lambda \geqslant n(r-1)$ and $f=\left\{f_{j}\right\}$ with $f_{j}$ the characteristic function of $Q_{j}$, then $S_{\lambda}(x)$ is bounded by a multiple of $\left|f^{*}(x)\right|_{r}^{r}$. Thus we have the following theorem. 
THeorem 4.1. Let $1<r<\infty, \lambda \geqslant n(r-1)$ and suppose $E$ is a set of finite measure with $\bigcup_{1}^{\infty} Q_{j} \subset E$. $a>0$

(a) If $q \geqslant 1 / r$ and $\omega \in A_{r q}$, there is a constant $O_{\alpha, r, \lambda}$ such that for all

$$
\omega\left(\left\{x \in \boldsymbol{R}^{n}: S_{\lambda}(x)>a\right\}\right) \leqslant C_{\alpha, r, \lambda} \alpha^{-a} \omega\left(\bigcup_{1}^{\infty} Q_{j}\right)
$$

(b) If $q>1 / r$ and $\omega \in A_{r q}$, there is a constant $C_{\alpha, r, \lambda}$ such that

$$
\int_{\boldsymbol{n}^{n}}\left|S_{\lambda}(x)\right|^{q} \omega(x) d x \leqslant C_{q, r, \lambda} \omega\left(\bigcup_{1}^{\infty} Q_{j}\right)
$$

(c) If $\omega(x)^{\alpha}$ is integrable on $E$ for some $q>1$, in particular if $\omega \in A_{\infty}$ and $E$ is a oube, then $S_{\lambda} \in \exp L_{\omega}(E)$.

For related results, sce A. P. Calderón [3], M. Kaneko and S. Yano [11].

\$5. Singular integrals. Let $K(x)$ be a convolution kernel satisfying the conditions

$$
\begin{array}{ll}
\text { (5.1) } & |K(x)| \leqslant B|x|^{-n}, \quad|\hat{K}(x)| \leqslant B \\
\text { and } & |K(x-y)-K(x)| \leqslant \theta(|y| /|x|)|x|^{-n} \text { for }|x| \geqslant 2|y| \\
(5.2) & \mid K(x)
\end{array}
$$

where $B$ is a constant and $\theta(t)$ is non-decreasing for $t>0, \theta(2 t) \leqslant c \theta(t)$ and satisfics the Dini condition

$$
\int_{0}^{1} \frac{\theta(t)}{t} d t<\infty .
$$

In particular, we may take $K(x)=\Omega(x) /|x|^{n}$ a Calderón-Zygmund kernel, i.o. $\Omega(x)$ homogeneous of degree zero, of mean value zero on the unit sphere in $\boldsymbol{R}^{n}$ and satisfying the Dini condition.

$$
\int_{0}^{1}(\delta(t) / t) d t<\infty
$$

where

$$
\delta(t)=\sup \{|\Omega(x)-\Omega(y)|:|x|=|y|=1,|x-y| \leqslant t\} .
$$

Define $T$ and $T^{*}$ by

$$
T f(x)=\text { P.V. } \int_{\boldsymbol{R}^{n}} K(x-y) f(y) d y,
$$

$$
T^{*} f(x)=\sup _{Q_{x}}\left\{\left.\right|_{\boldsymbol{n}^{n}-Q_{x}} K(x-y) f(y) d y \mid: Q_{x} \text { a cube contered at } x\right\} .
$$

M. Kaneko and S. Yano [11] have shown that $\omega \in A_{p}$ implies the inequalities

$$
\int_{R^{n}}\left|T^{*} f(x)\right|^{p} \omega(x) d x \leqslant C_{p} \int_{\mathbb{R}^{n}}|f(x)|^{p} \omega(x) d x \quad(1<p<\infty)
$$

and

$$
\text { (5.5) } \quad \omega\left(\left\{x \in \boldsymbol{R}^{n}: T^{*} f(x)>\alpha\right\}\right) \leqslant C \alpha^{-1} \int_{\boldsymbol{R}^{n}}|f(x)| \omega(x) d x \quad(p=1) .
$$

Earlier, R. Coifman and C. Fefferman [5] proved (5.4) for a more restricted class of kernels, namely when $\theta(t)=B t$ in (5.2). Of course (5.4) and (5.5) yield the corresponding inequalities for $T$ in place of $T^{*}$. Unweighted vector analogues of (5.4) for $T$ have been given by A. Cordoba and C. Fefferman [6], see also A. Benedek, A. Calderón and R. Panzone [1], J. Marcinkiewicz and A. Zygmund [12].

Suppose now that $\left\{K_{k}(x)\right\}$ is a sequence of convolution kernels satisfying (5.1)-(5.3) with a uniform constant $B$ and fixed $\theta$ independent of $k$. If $f=\left\{f_{k}\right\}$, let $T f=\left\{T_{k} f_{k}\right\}, T^{*} f=\left\{T_{k}^{*} f_{k}\right\}$ where of course $T_{k}$ and $T_{k}^{*}$ are the operators defined above corresponding to the kernel $K_{k}$. We shall prove the following vector-valued analogues of (5.4) and (5.5):

THEOREM 5.1. Let $1<r<\infty$ and suppose $\omega \in A_{1}$. There exists a constant $C_{r}$ such that for all $\alpha>0$

$$
\omega\left(\left\{x \in \boldsymbol{R}^{n}:\left|T^{*} f(x)\right|_{r}>\alpha\right\}\right) \leqslant C_{r} \alpha^{-1} \int_{\boldsymbol{R}^{n}}|f(x)|_{r} \omega(x) d x .
$$

THEOREM 5.2. Let $1<r<\infty, 1<p<\infty$ and suppose $\omega \in A_{p}$. There is a constant $O_{r, p}$ such that

$$
\int_{\boldsymbol{R}^{n}}\left|T^{*} f(x)\right|_{r}^{p} \omega(x) d x \leqslant C_{r, p} \int_{\boldsymbol{R}^{n}}|f(x)|_{r}^{p} \omega(x) d x .
$$

Again (5.6) and (5.7) imply the corresponding results for $T$ in place of $T^{*}$, however, it is possible to give an alternate proof of those results in the special case $\theta(t)=B t$ by following the line of proof used for Theorem 3.1. In the course of such a proof, the Calderón-Zygmund decomposition is used to write $f=g+b$ (as in the proof of Theorem 5.1 below); the contribution of $T g$ is handled in the same way that $f^{\prime *}$ was while that of $T b$ is estimated as in [15], p. 32 by the Marcinkiewicz integral and an appeal to Theorem 4.1. The required duality relation is provided in A. Cordoba and C. Fefferman [6].

The proof of Theorem 5.2 requires the following result which is a consequence of Theorem 5.1. 
LEMaca 5.1. Let $1<r<\infty$ and suppose $\omega \in A_{\infty}$. There are constants $C_{r}, \delta>0$ such that

(5.8) $\quad \omega\left(\left\{x \in \boldsymbol{R}^{n}:\left|T^{*} f(x)\right|_{r}>2 \alpha,|f(\cdot)|_{r}^{*}(x) \leqslant \gamma \alpha,\left|f^{*}(x)\right|_{r} \leqslant \gamma \alpha\right\}\right)$

$$
\leqslant O_{r} \gamma^{\delta} \omega\left(\left\{x \in \boldsymbol{R}^{n}:\left|T^{*} f(x)\right|_{r}>\alpha\right\}\right)
$$

for all $a>0, \gamma>0$.

Proof of Theorem 5.1. Suppose $f \in S$ and $\alpha>0$. As in the proof of Theorem 3.1 the Calderón-Zygmund decomposition yields a sequence of disjoint cubes $\left\{Q_{j}\right\}$ and we write $f=g+b$ where $g=\left\{g_{k}\right\}$,

$$
g_{k}(x)=\left\{\begin{array}{lll}
f_{f_{k}}(x) & \text { if } & x \notin \Omega=\bigcup_{1}^{\infty} Q_{j}, \\
\frac{1}{\left|Q_{j}\right|} \int_{Q_{j}} f_{k}(y) d y & \text { if } & x \in Q_{j}, j=1,2, \ldots
\end{array}\right.
$$

Since $\omega \in A_{1}$, we have

$$
\begin{aligned}
\int_{Q_{j}}|g(x)|_{r} \omega(x) d x & \leqslant \frac{1}{\left|Q_{j}\right|} \int_{Q_{j}}|f(y)|_{r} d y \int_{Q_{j}} \omega(x) d x \\
& \leqslant\left(\frac{1}{\left|Q_{j}\right|} \int_{Q_{j}}|f(y)|_{r} \omega(y) d y\right)\left(\underset{x \in Q_{j}}{\operatorname{ess} \sup _{j}} \frac{1}{\omega(x)}\right) \int_{Q_{j}} \omega(x) d x \\
& \leqslant K \int_{Q_{j}}|f(y)|_{r} \omega(y) d y
\end{aligned}
$$

so that

(5.9)

$$
\int_{\boldsymbol{R}^{n}}|g(x)|_{r} \omega(x) d x \leqslant O \int_{\boldsymbol{R}^{n}}|f(x)|_{r} \omega(x) d x
$$

Thus, it suffices to prove

$$
\omega\left(\left\{x \in \boldsymbol{R}^{n}:\left|T^{*} g(x)\right|_{r}>\alpha\right\}\right) \leqslant O_{r} \alpha^{-1} \int_{\boldsymbol{R}^{n}}|g(x)|_{r} \omega(x) d x
$$

and

(5.11) $\quad \omega\left(\left\{x \in \boldsymbol{R}^{n}:\left|T^{*} b(x)\right|_{r}>\alpha\right\}\right) \leqslant O_{r} a^{-1} \int_{n_{n}}|f(x)|_{r} \omega(x) d x$.

Since $\omega \in A_{r}$ by (2.2), the Ohebysher inequality, (5.4) and the fact that $|g(x)|_{r} \leqslant 2^{n} \alpha$ yields $(5.10)$ immediately. Let $Q_{j}^{*}$ be the cube concentric with $Q_{j}$ with diameter $3 \sqrt{n}$ times as large. The same proof as that given in $[15]$, pp. 43-44, shows that for $x \notin \Omega^{*}=\bigcup_{1}^{\infty} Q_{j}^{*}$

$$
T_{k}^{*} b_{k}(x) \leqslant \sum_{j=1}^{\infty} \int_{Q_{j}}\left|K_{k}(x-y)-K_{k}\left(x-y_{j}\right)\right|\left|b_{k}(y)\right| d y+O b_{k}^{*}(x)
$$

where $y_{j}$ is the center of $Q_{j}$. Now for such $x$ and $y \in Q_{j}$ we have $|x-y| \geqslant 2\left|y-y_{j}\right|$ where $y_{j}$ ts

$$
\left|T^{*} b(x)\right|_{r} \leqslant \sum_{j=1}^{\infty} \int_{Q_{j}} \theta\left(\frac{\left|y-y_{j}\right|}{|x-y|}\right) \frac{|b(y)|_{r}}{|x-y|^{n}} d y+C\left|b^{*}(x)\right|_{r}
$$

for $x \notin \Omega^{*}$. Denoting the sum of terms on the right of (5.12) by $\Sigma(x)$, is suffices to show

$$
\omega\left(\left\{x \in R^{n}-\Omega^{*}: \Sigma(x)>\alpha\right\}\right) \leqslant C_{r} \alpha^{-1} \int_{\boldsymbol{R}^{n}}|b(x)|_{r} \omega(x) d x
$$

in view of Theorem 3.1 and the estimate, as in (3.9),

$$
\omega\left(\Omega^{*}\right) \leqslant C \omega(\Omega) \leqslant O \alpha^{-1} \int_{\mathbf{R}^{n}}|f(x)|_{r} \omega(x) d x .
$$

Now if $d_{j}=$ diameter $\left(Q_{j}\right)$, then

$$
\begin{aligned}
\int_{\boldsymbol{R}^{n}-\Omega^{*}} \Sigma(x) \omega(x) d x & \leqslant \sum_{j=1}^{\infty} \int_{Q_{j}}|b(y)|_{r} d y \int_{\boldsymbol{R}^{n}-Q_{j}^{*}} \theta\left(\frac{\left|y-y_{j}\right|}{|x-y|}\right) \frac{\omega(x)}{|x-y|^{n}} d x \\
& \leqslant \sum_{j=1}^{\infty} \int_{Q_{j}}|b(y)|_{r} d y \int_{|x-y|>a_{j}} \theta\left(\frac{d_{j}}{|x-y|}\right) \frac{\omega(x)}{|x-y|^{n}} d x
\end{aligned}
$$

and Theorem 2 of [15], pp. 62-63, shows that the inner integral is bounded by $C \omega^{*}(y)$, and since $\omega \in A_{1}$ implies $\omega^{*}(y) \leqslant C \omega(y)$ a.e. we have

$$
\int_{\mathbf{R}^{n}-\Omega^{*}} \Sigma(x) \omega(x) d x \leqslant C \int_{\Omega}|b(y)|_{r} \omega(y) d y
$$

which yields, by the Chebysher inequality, (5.13) as required. The proof of Theorem 5.1 is complete.

Proof of Lemma 5.1. We follow as closely as possible the proofs given in [5] and [11] for the scalar valued case. By the Whitney Lemma [15], given union of non-overp. 16, the open set $\Omega=\left\{x \in \boldsymbol{R}\right.$. $\left.\left|T^{*} f(x)\right|_{r}>\alpha\right\}$ is the union ${ }^{2}$ to $\boldsymbol{R}^{n}-\Omega$ lapping cubes $\left|Q_{j}\right|$ with the property that the distance from $Q_{j}$ to $\boldsymbol{R}^{n}-\Omega$ is comparable to $d_{j}=$ diameter $\left(Q_{j}\right)$. Thus there are points $x_{j} \in \boldsymbol{R}^{n}-\Omega$ such that the distance from $x_{j}$ to $Q_{j}$ is less than $4 d_{j}$. Let $\bar{Q}_{j}$ be the cube concentric with $Q_{j}$ but of diameter say, $(21 \sqrt{n}) d_{j}$. Note that $Q_{j} \subset \bar{Q}_{j}$. The main step in the proof is the inequality

(5.14) $\left|\left\{x \in Q_{j}:\left|T^{*} f(x)\right|_{r}>2 a,|f(\cdot)|^{*}(x) \leqslant \gamma \alpha,\left|f^{*}(x)\right|_{r} \leqslant \gamma \alpha\right\}\right| \leqslant C_{r} \gamma\left|Q_{j}\right|$

for then (5.8) follows by applying the definition of $\omega \in A_{\infty}$ and summing over $j$. 
To prove (5.14) we may assume that there are points $\xi_{j}$ and $\eta_{j}$ in $Q_{j}$ such that $|f(\cdot)|_{r}^{*}\left(\xi_{j}\right) \leqslant \gamma \alpha,\left|f^{*}\left(\eta_{j}\right)\right|_{r} \leqslant \gamma \alpha$ and also that $\gamma$ is small, otherwise the inequality is trivial. Write $f=u+v$ where $u=\left\{u_{k}\right\}, u_{k}(x)$ $=f_{k}(x) \chi \bar{Q}_{j}(x)$. Since $\xi_{j} \in Q_{j} \subset \bar{Q}_{j}$ we have

$$
\frac{1}{\left|\bar{Q}_{j}\right|} \int_{\boldsymbol{R}^{n}}|u(x)|_{r} d x=\frac{1}{\left|\bar{Q}_{j}\right|} \int_{\bar{Q}_{j}}|f(x)|_{r} d x \leqslant|f(\cdot)|_{r}^{*}\left(\xi_{j}\right) \leqslant \gamma \alpha
$$

and since the weight function $\omega(x) \equiv 1$ satisfies $A_{1}$, Theorem 5.1 shows that

$$
\text { (5.15) } \quad\left|\left\{x \in \boldsymbol{R}^{n}:\left|T^{*} u(x)\right|_{r}>\alpha / 2\right\}\right| \leqslant O_{r} \gamma\left|Q_{j}\right| \text {. }
$$

Now it is shown in [11], pp. 579-580, that for $x \in Q_{j}$ we have

and hence also

$$
T_{k}^{*} v_{k}(x) \leqslant T_{k}^{*} f_{k}\left(x_{j}\right)+O f_{k}^{*}\left(\eta_{j}\right)
$$

$$
\left|T^{*} v(x)\right|_{r} \leqslant\left|T^{*} f\left(x_{j}\right)\right|_{r}+O\left|f^{*}\left(\eta_{j}\right)\right|_{r} \leqslant \alpha+C_{\gamma \alpha}
$$

since $x_{j} \notin \Omega$. Hence (5.15) yields

$$
\left|\left\{x \in Q_{j}:\left|T^{*} f(x)\right|_{r}>\alpha / 2+\alpha+C_{\gamma} \alpha\right\}\right| \leqslant O_{r} \gamma\left|Q_{j}\right|
$$

which implies (5.14) for small $\gamma$ as required. The lemma is proved.

Proof of Theorem 5.2. It is sufficient to prove the inequality for $f=\left\{f_{k}\right\}$ with $f_{k}=0$ for all sufficiently large $k$, say $k>N$, for then the general case follows by the monotone convergence theorem. Since $\omega \in A_{p}$ implies $\omega \in A_{\infty}$, Lemma 5.1 shows that

$$
\begin{aligned}
\omega\left(\left\{x \in \boldsymbol{R}^{n}:\left|T^{*} f(x)\right|_{r}>\alpha\right\}\right) \leqslant \sigma_{r} \gamma^{\delta} \omega\left(\left\{x \in \boldsymbol{R}^{n}:\left|T^{*} f(x)\right|_{r}>\alpha / 2\right\}\right)+ \\
+\omega\left(\left\{x \in \boldsymbol{R}^{n}:|f(\cdot)|_{r}^{*}(x)>\gamma \alpha\right\}\right)+\omega\left(\left\{x \in \boldsymbol{R}^{n}:\left|f^{*}(x)\right|_{r}>\gamma \alpha\right\}\right) .
\end{aligned}
$$

Multiplying this by $p \alpha^{p-1}$ and integrating over $\alpha \in(0, \infty)$ yields

$$
\begin{aligned}
\int_{\boldsymbol{R}^{n}}\left|T^{*} f(x)\right|_{r}^{p} \omega(x) d x & \leqslant O_{r, p} \gamma^{\delta} \int_{\boldsymbol{R}^{n}}\left|T^{*} f(x)\right|_{r}^{p} \omega(x) d x+ \\
& +\left.\left.O_{\gamma, p} \int_{\boldsymbol{R}^{n}}|| f(\cdot)\right|_{r} ^{*}(x)\right|^{p} \omega(x) d x+C_{\gamma, p} \int_{\boldsymbol{R}^{n}}\left|f^{*}(x)\right|_{r}^{p} \omega(x) d x .
\end{aligned}
$$

By our assumption on $f$ and (5.4) we see that

$$
\int_{\mathbf{R}^{n}}\left|T^{*} f(x)\right|_{r}^{p} \omega(x) d x \leqslant \sigma_{p}\left(\sum_{k=1}^{N}\left(\int_{\mathbf{R}^{n}}\left|f_{k}(x)\right|^{p} \omega(x) d x\right)^{1 / p}\right)^{p}<\infty,
$$

so that upon choosing $\gamma$ such that $C_{r, p} \gamma^{\sigma} \leqslant 1 / 2$ we obtain the desired inequality from (1.5) and Theorem 3.1. The proof is complete.

\section{References}

11] A. Benedek, A. P. Calder ón and R. Panzone, Convolution operators on Banach space valued functions, Proc. Nat. Acad. Sc. U.S.A. 48 (1962), pp. 356-365. B. Benes and R. Panzone, The spaces $L^{p}$ with mixed norm, Duke Math. J. 28 (1961), pp. 301-324.

[3] A. P. Calderón, On an integral of Marcinkiewicz, Studia Math. 57 (1976), pp. 279-284.

[4] A. P. Calderón and A. Zygmund, A note on the interpolation of sublinear operations, Amer. J. Math. 78 (1956), pp. 282-288.

[5] R. R. Coifman and C. Fefferman, Weighted norm inequalities for maximal functions and singular integrals, Studia Math. 51 (1974), pp. 241-250.

6] $A$. Cordo and C. Fefferman, A weighted norm inequality for singular integrals, Studia Math. 57 (1976), pp. 97-101.

[7] C. Fefferman and E. M. Stein, Some maximal inequalities, Amer. J. Math. 93 (1971), pp. 107-115.

[8] H. P. Heinig, Weighted maximal inequalities for $l^{r}$-valued functions, Canad. Math. Bull. 19 (1976), pp. 445-453.

[9] R. A. Hunt, B. Muckenhoupt and R. L. Wheeden, Weighted norm inequalities for the conjugate function and Hilbert transform, Trans. Amer. Math. Soc. 176 (1973), pp. 227-251.

[10] $\mathrm{R}, \mathrm{T}$. John, Weighted norm inequalities for singular and hypersingular integrals, Thesis, Rutgers University, New Brunswick, N. J. 1975.

[11] M. Kaneko and S. Yano, Weighted norm inequalities for singular integrals, J. Math. Soc. Japan 27 (1975), pp. 570-588.

[12] J. Marcinkiewicz and A. Zygmund, Quelques inégalités pour les opérations lineaires, Fund. Math. 32 (1939), pp. 115-121.

[13] ] W The Hardy maximal function Trans. Amer. Math. Soc. 165 (1972), pp. 207-226.

[14] - The equivalence of two conditions for weight functions, Studia Math. 49 (1974), pp. 101-106.

[15] E. M. Stein, Singular integrals and differentiability properties of functions, Princeton Univ. Press, 1970.

[16] A. Z mation Theory 2 (1969), pp. 249-257.

[17]

\section{DEPARTMENT OF MATHEMATIO}

DARITERSTTY OF ALBERTA, EDMONTON, ALBERTA

PRUDENTIAL PROPHRTYY AND CASUALTY INSURANCE, CO.

HOLMDEL, NEW JERSEY 